\title{
INEQUALITIES FOR THE MAXIMAL EIGENVALUE OF A NONNEGATIVE MATRIX
}

\author{
by LINA YEH
}

(Received 1 February, 1996; revised 30 October, 1996)

\begin{abstract}
Two-sided bounds are obtained for the maximal eigenvalue of a positive matrix by iterating computations of row sums. The result provides an algorithm for approximating the maximal eigenvalue of a nonnegative matrix.
\end{abstract}

1. Introduction. An $n \times n$ real matrix $A=\left(a_{i j}\right)$ is called nonnegative $(A \geq 0)$ if all $a_{i j} \geq 0 ; A$ is positive $(A>0)$ if all $a_{i j}>0$. In 1907 Perron showed that every nonnegative matrix $A$ has an eigenvalue $r$ such that $r \geq|\lambda|$, for every eigenvalue $\lambda$ of $A$. The eigenvalue $r$ is called the maximal eigenvalue of $A$. Since then the theory of nonnegative matrices has developed and occurred in various parts of mathematics such as the Theory of Stochastic Process, numerical analysis and dynamic programming. It is well known (see, for example, [4]) that the maximal eigenvalue has bounds:

$$
\min _{i} r_{i} \leq r \leq \max _{i} r_{i}
$$

where $r_{i}=r_{i}(A)=\sum_{i=1}^{n} a_{i t}$. There have been a number of interesting papers on finding the bounds for the maximal eigenvalue of a nonnegative matrix ([1], [2], [5]). In this paper, we find two-sided bounds for the maximal eigenvalue of a nonnegative matrix by an Iterative algorithm. Furthermore, the algorithm can be used to estimate the maximal eigenvalue. Suppose that $A$ has positive row sums $r_{1}, \ldots, r_{n}$, and let $D=\operatorname{diag}\left(r_{1}, \ldots, r_{n}\right)$. Then the $i$ th row sum of the matrix $D^{-1} A D$ is $\frac{1}{r_{i}} \sum_{t=1}^{n} a_{t t} r_{t}$. Since $D^{-1} A D$ and $A$ have the same eigenvalues, we replace $A$ by $D^{-1} A D$ in inequality (1), and obtain

$$
\min _{i}\left(\frac{1}{r_{i}} \sum_{t=1}^{n} a_{i t} r_{t}\right) \leq r \leq \max _{i}\left(\frac{1}{r_{i}} \sum_{t=1}^{n} a_{i t} r_{t}\right)
$$

For any positive numbers $q_{1}, \ldots, q_{m}$ and real numbers $p_{1}, \ldots, p_{m}$, we have the inequality (see [3, p. 26])

$$
\min _{i} \frac{p_{i}}{q_{i}} \leq \frac{p_{1}+p_{2}+\ldots+p_{m}}{q_{1}+q_{2}+\ldots+q_{m}} \leq \max _{i} \frac{p_{i}}{q_{i}}
$$

Moreover, the equality holds on either side of (3) if and only if $p_{i} / q_{i}$ is constant for all $i=1,2, \ldots, m$. Suppose $A$ has positive row sums. Applying (3) to the right-hand side of (2), we obtain

$$
\max _{i}\left(\frac{1}{r_{i}} \sum_{t=1}^{n} a_{i t} r_{t}\right)=\max _{i} \frac{\sum_{t=1}^{n} a_{i t} r_{t}}{\sum_{t=1}^{n} a_{i t}} \leq \max _{i} r_{i}
$$

Glasgow Math. J. 39 (1997) 275-284. 
Similarly, we have $\min _{i} r_{i} \leq \min _{i}\left(\frac{1}{r_{i}} \sum_{t=1}^{n} a_{t t} r_{t}\right)$. Thus

$$
\min _{i} r_{i} \leq \min _{i}\left(\frac{1}{r_{i}} \sum_{t=1}^{n} a_{i t} r_{t}\right) \leq r \leq \max _{i}\left(\frac{1}{r_{i}} \sum_{t=1}^{n} a_{i t} r_{t}\right) \leq \max _{i} r_{i} \text {. }
$$

Hence (2) gives a sharper bound for $r$ than (1).

We shall iterate the process described in (2), and obtain sequences of two-sided bounds for the maximal eigenvalue. Furthermore, we show that the sequences converge to the maximal eigenvalue for a positive matrix in Theorem 4 , and nonnegative matrix of the type described in Theorem 5 .

2. Preliminaries. Let $A \in M_{n}$ be a nonnegative matrix with positive row sums. For convenience, we give some symbols and definitions. First, we initialize $A^{(0)}=A=\left(a_{i j}^{(0)}\right)$, $r_{i}^{(0)}=\sum_{j=1}^{n} a_{i j}^{(0)}$, and $r^{(0)}=\left[r_{1}^{(0)} r_{2}^{(0)} \ldots r_{n}^{(0)}\right]^{t}$. Moreover, set $D^{(0)}=D$. We define, by induction, the diagonal matrices $D^{(k)}$ with the $(i, i)$ th entry being the $i$ th row sum of the matrix

$$
\left(D^{(k-1)}\right)^{-1}\left(D^{(k-2)}\right)^{-1} \ldots\left(D^{(0)}\right)^{-1} A D^{(0)} \ldots D^{(k-2)} D^{(k-1)},
$$

for $k=1,2, \ldots$ Next, for $k=0,1,2, \ldots$, set the recurrence

$$
A^{(k+1)}=\left(D^{(k)}\right)^{-1} A^{(k)} D^{(k)} \equiv\left(a_{i j}^{(k+1)}\right)
$$

Then

$$
\left(D^{(k)}\right)^{-1} A^{(k)}=\left(\frac{a_{i j}^{(k)}}{r_{i}^{(k)}}\right)
$$

and

$$
A^{(k+1)}=\left(a_{i j}^{(k)} \frac{r_{j}^{(k)}}{r_{i}^{(k)}}\right)
$$

where $r_{i}^{(k)}=\sum_{j=1}^{n} a_{i j}^{(k)}$. Let $r^{(k)}=\left[\begin{array}{lll}r_{1}^{(k)} & r_{2}^{(k)} \ldots & r_{n}^{(k)}\end{array}\right]^{t}$. By (7) we have

$$
r_{i}^{(k+1)}=\sum_{i=1}^{n} a_{i t}^{(k+1)}=\sum_{i=1}^{n} a_{i t}^{(k)} \frac{r_{i}^{(k)}}{r_{i}^{(k)}}
$$

By (6), the vector form of (8) becomes

$$
r^{(k+1)}=\left(D^{(k)}\right)^{-1} A^{(k)} r^{(k)} \quad(k=0,1,2, \ldots) .
$$

Two matrices $A=\left(a_{i j}\right)$ and $B=\left(b_{i j}\right)$ of the same size are said to have the same zero pattern if $a_{i j}=0$ whenever $b_{i j}=0$. As a consequence of (6) and (7), the matrices $A, A^{(k)}$, and $\left(D^{(k)}\right)^{-1} A^{(k)}(k=0,1,2, \ldots)$ all have the same zero pattern.

The next result expresses the row sums of the iterative matrix $A^{(k)}$ in terms of the row sums of $A^{k}$.

THEOREM 1. Let $A \in M_{n}$ be a nonnegative matrix with nonzero row sums. Then for 
every $i=1,2, \ldots, n, r_{i}^{(k)}=r_{i}\left(A^{k+1}\right) / r_{i}\left(A^{k}\right)(k=1,2, \ldots)$ and the sequence $\left\{\max _{i} r_{i}^{(k)}\right\}_{k}$ is decreasing, the sequence $\left\{\min _{i} r_{i}^{(k)}\right\}_{k}$ is increasing and both are convergent, where $r_{i}\left(A^{k}\right)$ is the ith row sum of the matrix $A^{k}$.

Proof. The proof is by induction on $k$. Suppose that $k=1$. Then by (8) we have

$$
r_{i}^{(1)}=\frac{\sum_{i} a_{i t} \sum_{j} a_{i j}}{r_{i}^{(0)}}=\frac{r_{i}\left(A^{2}\right)}{r_{i}(A)} .
$$

Suppose that the assertion is true for $k \leq m$. From (5) and (8),

$$
r_{i}^{(m+1)}=r_{i}\left(A^{(m+1)}\right)=r_{i}\left(\left(D^{(m)}\right)^{-1} A^{(m)} D^{(m)}\right)=\sum_{i=1}^{n} a_{i t}^{(m)} \frac{r_{t}^{(m)}}{r_{i}^{(m)}},
$$

where $D^{(m)}=\operatorname{diag}\left(r_{1}^{(m)}, r_{2}^{(m)}, \ldots, r_{n}^{(m)}\right)$. By (5) we have

$$
A^{(m)}=\left(D^{(m-1)}\right)^{-1}\left(D^{(m-2)}\right)^{-1} \ldots\left(D^{(0)}\right)^{-1} A D^{(0)} \ldots D^{(m-2)} D^{(m-1)} .
$$

Then $a_{i t}^{(m)}=\left(r_{i}^{(m-1)}\right)^{-1}\left(r_{i}^{(m-2)}\right)^{-1} \ldots\left(r_{i}^{(0)}\right)^{-1} a_{i t} r_{i}^{(0)} \ldots r_{i}^{(m-2)} r_{i}^{(m-1)}$. By the induction hypothesis, we compute that

$$
\begin{aligned}
r_{i}^{(m+1)} & =\frac{\sum_{i=1}^{n} a_{i t}^{(m)} r_{t}^{(m)}}{r_{i}^{(m)}} \\
& =\frac{\sum_{t}\left(r_{i}^{(m-1)}\right)^{-1}\left(r_{i}^{(m-2)}\right)^{-1} \ldots\left(r_{i}^{(0)}\right)^{-1} a_{i r} r_{t}^{(0)} \ldots r_{t}^{(m-2)} r_{t}^{(m-1)} r_{i}^{(m)}}{r_{i}^{(m)}} \\
& =\frac{\sum_{t} \frac{r^{\prime}\left(A^{m-1}\right)}{r_{i}\left(A^{m}\right)} \frac{r_{i}\left(A^{m-2}\right)}{r_{i}\left(A^{m-1}\right)} \cdots \frac{1}{r_{i}(A)} a_{i t} r_{t}(A) \ldots \frac{r_{t}\left(A^{m-1}\right)}{r_{t}\left(A^{m-2}\right)} \frac{r_{t}\left(A^{m}\right)}{r_{t}\left(A^{m-1}\right)} \frac{r_{t}\left(A^{m+1}\right)}{r_{t}\left(A^{m}\right)}}{\frac{r_{i}\left(A^{m+1}\right)}{r_{i}\left(A^{m}\right)}} \\
& =\frac{\sum_{t} a_{i t} r_{t}\left(A^{m+1}\right)}{r_{i}\left(A^{m+1}\right)}=\frac{r_{i}\left(A^{m+2}\right)}{r_{i}\left(A^{m+1}\right)} .
\end{aligned}
$$

This completes the induction. Next, by inequality (3), we have

$$
r_{t}^{(k+1)}=\frac{r_{i}\left(A^{k+2}\right)}{r_{i}\left(A^{k+1}\right)}=\frac{\sum_{i} a_{i i} r_{t}\left(A^{k+1}\right)}{\sum_{t} a_{i t} r_{t}\left(A^{k}\right)} \leq \max _{t} \frac{r_{t}\left(A^{k+1}\right)}{r_{t}\left(A^{k}\right)}=\max _{t} r_{i}^{(k)}
$$


It follows that $\max _{i} r_{i}^{(k+1)} \leq \max _{i} r_{i}^{(k)}$. The sequence $\left\{\max _{i} r_{i}^{(k)}\right\}_{k}$ is then decreasing and thus convergent. The proof that the sequence $\left\{\min _{i} r_{i}^{(k)}\right\}_{k}$ is increasing is similar.

The iterative matrix $A^{(k+1)}$ in (5) is similar to $A$; it has the same maximal eigenvalue as $A$ and hence

$$
\min _{i} r_{i}^{(k+1)} \leq r \leq \max _{i} r_{i}^{(k+1)}
$$

Taking the limit on both sides of (10), we obtain

$$
\lim _{k \rightarrow \infty} \min _{i} r_{l}^{(k+1)} \leq r \leq \lim _{k \rightarrow \infty} \max _{i} r_{i}^{(k+1)}
$$

Two matrices $A$ and $B$ are permutationally equivalent if there exists a permutation matrix $P$ such that $B=P^{\prime} A P$. We show in the following that the inequalities (10) and (11) are invariant under permutation equivalence.

THEOREM 2. Let $A, B \in M_{n}$ be permutationally equivalent nonnegative matrices with nonzero row sums. Then, for $k=0,1,2, \ldots$, we have

$$
\max _{i} r_{i}^{(k)}(A)=\max _{i} r_{i}^{(k)}(B) \text { and } \min _{i} r_{i}^{(k)}(A)=\min _{i} r_{i}^{(k)}(B),
$$

where $r_{i}^{(k)}(A)$ and $r_{i}^{(k)}(B)$ are the ith row sums of $A^{(k)}$ and $B^{(k)}$ respectively.

Proof. Suppose $B=P^{t} A P$, for some permutation matrix $P$. Then, for every $i=1,2, \ldots, n$, there exists $j$ such that $r_{i}(B)=r_{j}(A)$. The index $j$ is determined by the permutation $P$. By Theorem 1 we have

$$
\begin{aligned}
\max _{i} r_{i}^{(k)}(B) & =\max _{i} r_{i}^{(k)}\left(P^{t} A P\right)=\max _{i} \frac{r_{i}\left(\left(P^{t} A P\right)^{k+1}\right)}{r_{i}\left(\left(P^{t} A P\right)^{k}\right)} \\
& =\max _{i} \frac{r_{i}\left(P^{t} A^{k+1} P\right)}{r_{i}\left(P^{\prime} A^{k} P\right)}=\max _{j} \frac{r_{j}\left(A^{k+1}\right)}{r_{j}\left(A^{k}\right)}=\max _{j} r_{j}^{(k)}(A) .
\end{aligned}
$$

The minimum can be proved similarly.

3. Approximation of the maximal eigenvalue. The inequality (11) gives two-sided bounds for the maximal eigenvalue. the question arises if the equality holds in (11). First we give a positive lower bound for nonzero entries of $\left(D^{(k)}\right)^{-1} A^{(k)}$.

THEOREM 3. Let $A \in M_{n}$ be a nonnegative matrix and let $q$ be a given positive integer. If $A$ has $q$ positive columns and $n-q$ zero columns, then there exists a positive number $d$ with $d \leq 1 / q$ such that every nonzero entry of $\left(D^{(k)}\right)^{-1} A^{(k)}$ is not less than $d$, for all $k=0,1,2, \ldots$.

Proof. The proof will be by induction on $k$. Let $r_{M}^{(k)}$ denote the maximum row sum of $A^{(k)}, m^{(0)}$ the minimum of nonzero entries of $A^{(0)}, m l^{(0)}$ the minimum of nonzero entries of the $l$ th column of $A^{(0)}$, and $M^{(0)}$ the maximum of nonzero entries of the $l$ th column of 
$A^{(0)}$. Set $\alpha=\min \frac{m_{l}^{(0)}}{M_{l}^{(0)}}$, where $l$ runs over all $q$ positive columns of $A$. We prove first, by induction, that for every nonzero entry $a_{i j}^{(k)}$,

$$
a_{i j}^{(k)} \min _{t} \frac{a_{j t}^{(k)}}{a_{i t}^{(k)}} \geq m^{(0)} \alpha \quad(k=0,1,2, \ldots)
$$

where the minimum $t$ is taken over all indices of positive columns of $A^{(k)}$.

It is trivial that the induction hypothesis holds when $k=0$. Suppose the induction is true for $k$. If $a_{i j}^{(k+1)}$ is nonzero, then $a_{i j}^{(k)}$ is nonzero. By (7) we have

This proves the assertion.

$$
a_{i j}^{(k+1)} \min _{t} \frac{a_{j i}^{(k+1)}}{a_{i t}^{(k+1)}}=a_{i j}^{(k)} \frac{r_{i}^{(k)}}{r_{i}^{(k)}} \min _{t} \frac{a_{i t}^{(k)} \frac{r_{t}^{(k)}}{r_{j}^{(k)}}}{a_{i t}^{(k)} \frac{r_{i}^{(k)}}{r_{i}^{(k)}}}=a_{i j}^{(k)} \min _{t} \frac{a_{j i}^{(k)}}{a_{i t}^{(k)}} \geq m^{(0)} \alpha .
$$

Suppose now that $a_{i j}^{(k)}$ is nonzero. From (7) and (8), we deduce that

$$
\begin{aligned}
a_{i j}^{(k)} & =a_{i j}^{(k-1)} \frac{r_{j}^{(k-1)}}{r_{i}^{(k-1)}}=a_{i j}^{(k-2)} \frac{r_{j}^{(k-2)}}{r_{i}^{(k-2)}} \frac{\sum_{t} a_{j i}^{(k-2)} \frac{r_{i}^{(k-2)}}{r_{j}^{(k-2)}}}{\sum_{t}^{(k-2)} a_{t}^{(k-2)}} r_{i}^{(k-2)} \\
& =a_{i j}^{(k-2)} \frac{\sum_{t} a_{j t}^{(k-2)} r_{i}^{(k-2)}}{\sum_{t} a_{i t}^{(k-2)} r_{t}^{(k-2)}} .
\end{aligned}
$$

Since $A$ and $A^{(k-2)}$ have the same zero pattern, by the hypothesis, both the $i$ th row and $j$ th row of $A^{(k-2)}$ have exactly $q$ nonzero entries that locate on the same columns. Thus the sums in the denominator and numerator of (12) have $q$ nonzero terms with the same $t$ indices. Apply those nonzero $q$ terms to the inequality (3). We obtain

$$
a_{i j}^{(k)} \geq a_{i j}^{(k-2)} \min _{i} \frac{a_{j i}^{(k-2)}}{a_{i t}^{(k-2)}} \geq m^{(0)} \alpha,
$$

where the minimum runs over those $q$ indices. This proves that every nonzero entry of $A^{(k)}$ is not less than $m^{(0)} \alpha$, for all $k=0,1,2, \ldots$

Finally, for any nonzero entry of $\left(D^{(k)}\right)^{-1} A^{(k)}$, we obtain

$$
\left(D^{(k)}\right)^{-1} A_{i j}^{(k)}=\frac{a_{i j}^{(k)}}{r_{i}^{(k)}} \geq \frac{m^{(0)} \alpha}{r_{M}^{(k)}} \geq \frac{m^{(0)} \alpha}{r_{M}^{(0)}} .
$$

The last inequality follows from the fact that the sequence $\left\{r_{M}^{(k)}\right\}_{k}$ is decreasing by Theorem 1. Define the positive number $d=\frac{m^{(0)} \alpha}{r_{M}^{(0)}}$. Since the $i$ th row of $A$ has $q$ nonzero elements, we have

$$
1=\sum_{i=1}^{n} \frac{a_{i t}^{(0)}}{r_{i}^{(0)}} \geq q \frac{m^{(0)} \alpha}{r_{M}^{(0)}}=q d .
$$

Thus $d \leq 1 / q$, and the proof is complete. 
Suppose that $A \in M_{n}$ is positive. Then the hypotheses of Theorem 3 are obviously satisfied. Define the iterative matrix

$$
P^{(k+1)}=\left(D^{(k)}\right)^{-1} A^{(k)} P^{(k)} \quad(k=1,2, \ldots),
$$

where $P^{(1)}=\left(D^{(0)}\right)^{-1} A^{(0)}$. We obtain the following approximation.

Theorem 4. Let $A \in M_{n}(n \geq 2)$ be a positive matrix. Then $P^{(k)}$ converges to a matrix

$$
S=\left(\begin{array}{cccc}
c_{1} & c_{2} & \ldots & c_{n} \\
c_{1} & c_{2} & \ldots & c_{n} \\
\vdots & \vdots & \ddots & \vdots \\
c_{1} & c_{2} & \ldots & c_{n}
\end{array}\right),
$$

for some positive numbers $c_{1}, c_{2}, \ldots, c_{n}$, and

$$
r=\lim _{k \rightarrow \infty} \max _{i} r_{i}^{(k)}=\lim _{k \rightarrow \infty} \min _{i} r_{i}^{(k)}=\lim _{k \rightarrow \infty} r_{i}^{(k)}=\sum_{i=1}^{n} c_{i} r_{i}^{(0)}
$$

Proof. For $k=1,2, \ldots$ we have from (13)

$$
P^{(k+1)}=\left(D^{(k)}\right)^{-1} A^{(k)}\left(D^{(k-1)}\right)^{-1} A^{(k-1)} \ldots\left(D^{(1)}\right)^{-1} A^{(1)}\left(D^{(0)}\right)^{-1} A^{(0)} .
$$

Since $\left(D^{(k)}\right)^{-1} A^{(k)}$ is positive, it follows from (14), that the matrix $P^{(k+1)}$ is positive. Let $m_{1}^{(k)}$ and $M_{1}^{(k)}$ denote respectively the smallest and the largest elements of the first column of $P^{(k)}$. We show first that for $k=1,2, \ldots$

$$
m_{1}^{(k+1)} \geq M_{1}^{(k)} d+m_{1}^{(k)}(1-d) \quad \text { and } \quad M_{1}^{(k+1)} \leq m_{1}^{(k)} d+M_{1}^{(k)}(1-d) .
$$

Let $P^{(k+1)}=\left(p_{i j}^{(k+1)}\right)$ and $\left(D^{(k)}\right)^{-1} A^{(k)}=\left(b_{i j}^{(k)}\right)$. By equation $(6)$, we have $\sum_{j=1}^{n} b_{i j}^{(k)}=1$, and by Theorem 3 , we have every entry $b_{i j}^{(k)} \geq d$ for some positive number $d \leq 1 / n$. For simplicity, assume that $m_{1}^{(k)}=p_{1}^{(k)}$ and $M_{1}^{(k)}=p_{21}^{(k)}$. Computing the first column entry of the matrix (13), we obtain

$$
\begin{aligned}
p_{i 1}^{(k+1)} & =\sum_{j=1}^{n} b_{i j}^{(k)} p_{j 1}^{(k)} \\
& =b_{i 1}^{(k)} p_{11}^{(k)}+\left(1-b_{i 1}^{(k)}-b_{i 3}^{(k)}-\ldots-b_{i n}^{(k)}\right) p_{21}^{(k)}+b_{i 3}^{(k)} p_{31}^{(k)}+\ldots+b_{i n}^{(k)} p_{n 1}^{(k)} \\
& =M_{1}^{(k)}-b_{i 1}^{(k)}\left(M_{1}^{(k)}-p_{11}^{(k)}\right)-b_{i 3}^{(k)}\left(M_{1}^{(k)}-p_{31}^{(k)}\right)-\ldots-b_{i n}^{(k)}\left(M_{1}^{(k)}-p_{n 1}^{(k)}\right) \\
& \leq M_{1}^{(k)}-b_{i 1}^{(k)}\left(M_{1}^{(k)}-p_{11}^{(k)}\right) \\
& =M_{1}^{(k)}-b_{i 1}^{(k)}\left(M_{1}^{(k)}-m_{1}^{(k)}\right) \\
& \leq M_{1}^{(k)}-d\left(M_{1}^{(k)}-m_{1}^{(k)}\right) \\
& =m_{1}^{(k)} d+(1-d) M_{1}^{(k)} .
\end{aligned}
$$


Hence $M_{1}^{(k+1)} \leq m_{1}^{(k)} d+(1-d) M_{1}^{(k)}$, and this proves the second part of (15). On the other hand

$$
\begin{aligned}
p_{i 1}^{(k-1)} & =\sum_{j=1}^{n} b_{i j}^{(k)} p_{i 1}^{(k)} \\
& =\left(1-b_{i 2}^{(k)}-b_{i 3}^{(k)}-\ldots-b_{i n}^{(k)}\right) p_{11}^{(k)}+b_{i 2}^{(k)} p_{21}^{(k)}+b_{i 3}^{(k)} p_{31}^{(k)}+\cdots+b_{i n}^{(k)} p_{n 1}^{(k)} \\
& =m_{1}^{(k)}+b_{i 2}^{(k)}\left(p_{21}^{(k)}-m_{1}^{(k)}\right)+b_{i 3}^{(k)}\left(p_{31}^{(k)}-m_{1}^{(k)}\right)+\ldots+b_{i n}^{(k)}\left(p_{n 1}^{(k)}-m_{1}^{(k)}\right) \\
& \geq m_{1}^{(k)}+b_{i 2}^{(k)}\left(p_{21}^{(k)}-m_{1}^{(k)}\right) \\
& =m_{1}^{(k)}+b_{i 2}^{(k)}\left(M_{1}^{(k)}-m_{1}^{(k)}\right) \\
& \geq m_{1}^{(k)}+d\left(M_{1}^{(k)}-m_{1}^{(k)}\right) \\
& =M_{1}^{(k)} d+(1-d) m_{1}^{(k)} .
\end{aligned}
$$

Thus $m_{1}^{(k+1)} \geq M_{1}^{(k)} d+(1-d) m_{1}^{(k)}$, and the first part of (15) is proved. Now by (15), we have $M_{1}^{(k-1)}-m_{1}^{(k+1)} \leq(1-2 d)\left(M_{1}^{(k)}-m_{1}^{(k)}\right)$. and thus

$$
M_{1}^{(k+1)}-m_{1}^{(k+1)} \leq(1-2 d)^{k}\left(M_{1}^{(1)}-m_{1}^{(1)}\right) \text {. }
$$

Hence $M_{1}^{(k+1)}-m_{1}^{(k+1)} \rightarrow 0$ as $k \rightarrow \infty$. Notice that $0 \leq 1-2 d<1$, by Theorem 3. Furthermore, by Theorem 1, the sequence $\left\{M_{1}^{(k)}\right\}_{k}$ decreases and $\left\{m_{1}^{(k)}\right\}_{k}$ increases. Therefore both sequences converge to the same limit, and thus the first column of $P^{(k)}$ converges to a column of the form $\left[\begin{array}{lllll}c_{1} & c_{1} & \ldots & c_{1}\end{array}\right]^{t}$, for some positive number $c_{1}$.

Applying the same argument to the remaining columns of $P^{(k)}$, we conclude that $P^{(k)}$ converges to a matrix

$$
S=\left(\begin{array}{cccc}
c_{1} & c_{2} & \ldots & c_{n} \\
c_{1} & c_{2} & \ldots & c_{n} \\
\vdots & \vdots & \ddots & \vdots \\
c_{1} & c_{2} & \vdots & c_{n}
\end{array}\right),
$$

for some positive numbers $c_{1}, c_{2}, \ldots, c_{n}$. From the recurrence relation (9), we obtain

$$
r^{(k)}=P^{(k)} r^{(0)}
$$

Then $\left\{r^{(k)}\right\}$ converges to $S r^{(0)}$, and hence for each $i,\left\{r_{i}^{(k)}\right\}_{k}$ converges to $r=c_{1} r_{1}^{(0)}+$ $c_{2} r_{2}^{(0)}+\ldots+c_{n} r_{n}^{(0)}$. This completes the proof.

We reduce the positivity in Theorem 4 to certain pattern of matrices, and obtain the following weaker version of Theorem 4.

THEOREM 5. Let $A \in M_{n}$ be a nonnegative matrix and let $q$ be a given positive integer. If $A$ is permutationally equivalent to a matrix with $q$ positive columns and $n-q$ zero columns, then

$$
r=\lim _{k \rightarrow \infty} \max _{i} r_{i}^{(k)}=\lim _{k \rightarrow \infty} \min _{i} r_{i}^{(k)}=\lim _{k \rightarrow \infty} r_{i}^{(k)} .
$$

Proof. By Theorem 2 we may assume the positive columns of $A$ appear in the first $q$ columns and zeros elsewhere. Let $P^{(k+1)}$ be the matrix defined in (14). Now $A$ and $\left(D^{(k)}\right)^{-1} A^{(k)}$ have the same zero pattern; i.e., the first $q$ columns of $\left(D^{(k)}\right)^{-1} A^{(k)}$ are positive and other columns are zeros. Thus the first $q$ columns of $P^{(k+1)}$ are positive and other columns are zeros. Suppose that $q \geq 2$. Apply the arguments of the proof in 
Theorem 4 to the first column of $P^{(k)}$. We obtain the inequality (16) with $0 \leq 1-2 d<1$. Repeating this process to the next $q-1$ columns of $P^{(k)}$, we see that the first $q$ columns of $P^{(k)}$ converge to a matrix

$$
\left(\begin{array}{cccc}
c_{1} & c_{2} & \ldots & c_{q} \\
c_{1} & c_{2} & \ldots & c_{q} \\
\vdots & \vdots & \ddots & \vdots \\
c_{1} & c_{2} & \ldots & c_{q}
\end{array}\right) \in M_{n \times q}
$$

for some positive numbers $c_{1}, c_{2}, \ldots, c_{q}$. Since the last $n-q$ columns of $P^{(k)}$ are zeros, it follows that, as $k \rightarrow \infty, P^{(k)}$ converges to

$$
s=\left(\begin{array}{ccccccc}
c_{1} & c_{2} & \ldots & c_{q} & 0 & \ldots & 0 \\
c_{1} & c_{2} & \ldots & c_{q} & 0 & \ldots & 0 \\
\vdots & \vdots & \ddots & \vdots & \vdots & \ddots & \vdots \\
c_{1} & c_{2} & \ldots & c_{q} & 0 & \ldots & 0
\end{array}\right) \in M_{n}
$$

By (17) we conclude that $\left\{r^{(k)}\right\}$ converges to $S r^{(0)}$, hence for each $i,\left\{r_{i}^{(k)}\right\}_{k}$ converges to $r=c_{1} r_{1}^{(0)}+c_{2} r_{2}^{(0)}+\ldots+c_{q} r_{q}^{(0)}$, and the conclusion follows. When $q=1$, it is clear that $a_{11}$ and 0 are the eigenvalues of $A$ and so $r=a_{11}$. By (5) we have

$$
A^{(1)}=\left(D^{(0)}\right)^{-1} A^{(0)} D^{(0)}=\left(\begin{array}{cccc}
a_{11} & 0 & \ldots & 0 \\
a_{11} & 0 & \ldots & 0 \\
\vdots & \vdots & \ddots & \vdots \\
a_{11} & 0 & \ldots & 0
\end{array}\right) \text {. }
$$

It follows that $A^{(k)}=A^{(1)}$ for all $k=2,3, \ldots$ Therefore $r_{i}^{(k)}=a_{11}$, and thus $r=a_{11}=$ $\lim _{k \rightarrow \infty} r_{i}^{(k)}$ for all $i$, completing the proof.

Theorem 4 provides an Iterative algorithm for approximating the maximal eigenvalue of a positive matrix. In general, let $A=\left(a_{i j}\right) \in M_{n}$ be a nonnegative matrix, and $r$ be the maximal eigenvalue of $A$. Given a positive number $\epsilon$, define a matrix $A_{\epsilon} \in M_{n}$ as follows:

$$
\left(A_{\epsilon}\right)_{i j}= \begin{cases}a_{i j}, & \text { if } a_{i j}>0 \\ \epsilon, & \text { if } a_{i j}=0\end{cases}
$$

Then $A_{\epsilon}$ is positive, and the maximal eigenvalue $r_{\varepsilon}$ of $A_{\epsilon}$ is estimated according to Theorem 4. Since eigenvalues are continuous functions of entries of the matrix, it follows that $A_{\varepsilon} \rightarrow A$ and $r_{\epsilon} \rightarrow r$ as $\epsilon \rightarrow 0$. Hence, if $\epsilon$ is small enough then an estimation of $r$ can be achieved by computations of $r_{e}$. We summarize the Iterative algorithm.

STEP 1. Initialize.

Let $k=0, A^{(0)}=A=\left(a_{i j}^{(0)}\right), r_{i}^{(0)}=\sum_{j=1}^{n} a_{i j}^{(0)}$ for $i=1,2, \ldots, n$ and $\delta=$ tolerance.

STEP 2. Find $\min$ and $\max$ row sums: $r_{m}^{(k)}=\min _{i} r_{i}^{(k)}$, and $r_{M}^{(k)}=\max _{i} r_{i}^{(k)}$.

STEP 3. Test. 
TABLE 1.

\begin{tabular}{cccc}
\hline Order. & Power method. & Iterative algorithm. & \multicolumn{1}{c}{ Matlab. } \\
\hline 100 & $12 / 4963 \cdot 6238537076$ & $12 / 4963 \cdot 6238537076$ & $4963 \cdot 6238537076$ \\
200 & $12 / 9881 \cdot 5030429984$ & $11 / 9881 \cdot 5030429984$ & $9881 \cdot 5030429984$ \\
300 & $11 / 14792 \cdot 7146278852$ & $11 / 14792 \cdot 7146278852$ & $14792 \cdot 7146278852$ \\
400 & $11 / 19719 \cdot 9743999372$ & $10 / 19719 \cdot 9743999372$ & $19719 \cdot 9743999372$ \\
500 & $10 / 24686 \cdot 1864859857$ & $10 / 24686 \cdot 1864859858$ & $24686 \cdot 1864859857$ \\
600 & $10 / 29632 \cdot 9292683024$ & $10 / 29632 \cdot 9292683025$ & $29632 \cdot 9292683024$ \\
700 & $10 / 34591 \cdot 5996879745$ & $10 / 34591 \cdot 5996879745$ & $34591 \cdot 5996879745$ \\
800 & $10 / 39545 \cdot 8711650438$ & $10 / 39545 \cdot 8711650439$ & $39545 \cdot 8711650439$ \\
900 & $10 / 44489 \cdot 6491412335$ & $10 / 44489 \cdot 6491412336$ & $44489 \cdot 6491412336$ \\
1000 & $10 / 49451 \cdot 5229094011$ & $9 / 49451 \cdot 5229094011$ & $49451 \cdot 5229094010$ \\
\hline
\end{tabular}

If $\left|r_{M}^{(k)}-r_{m}^{(k)}\right|<\delta$, then we obtain an approximation for the maximal eigenvalue or else $a_{i j}^{(k+1)}=a_{i j}^{(k)} r_{j}^{(k)} / r_{i}^{(k)}$ and $r_{i}^{(k+1)}=\sum_{j=1}^{n} a_{i j}^{(k+1)}, i=1,2, \ldots, n$.
Replace $k$ by $k+1$. Go to step 2.

The test condition Step 3 may be replaced by $\left|r_{M}^{(k+1)}-r_{M}^{(k)}\right|<\delta$ or $\left|r_{m}^{(k+1)}-r_{m}^{(k)}\right|<\delta$. We run MATLAB programs on the DEC Alpha Sever-2100, and list in Table 1 the numbers of iterations required to estimate the maximal eigenvalue by the Power method and the Iterative algorithm for nonnegative matrices of order 100 to 1000 with $\delta=1.0 \times 10^{-10}$. In Table 1 , there are two values in the Power method column and Iterative algorithm column. The first value is the number of iterations, the second value is the approximated maximal eigenvalue, and the Matlab software evaluates the maximal eigenvalue in the last column. The entries of a sample run matrix for the Iterative algorithm are generated by the following formula:

$$
\begin{aligned}
& A=\operatorname{rand}(n) \text {; } \\
& \text { if } A(i, j)<0 \cdot 1 \text {, then } A(i, j)=\text { eps } \approx 2 \cdot 2 \times 10^{-16}(A(i, j)=0 \text { for Power Method) } \\
& \text { or else } A(i, j)=A(i, j) \times 100 \text {. }
\end{aligned}
$$

From the experiment, the number of iterations required to approximate the maximal eigenvalue by the Iterative algorithm is close to that of the Power method with initial vector $x=[1]$. This similarity also happens to tridiagonal matrices and matrices whose maximal eigenvalue are relatively dense, such as the tridiagonal matrix $W_{21}^{+}=\left(w_{i j}\right) \in M_{21}$ (see $[6,5.45])$, where $w_{i l}=11-i(i=1, \ldots, 11), w_{i i}=i-11(i=12, \ldots, 21)$ and $w_{i i+1}=$ $w_{i+1 i}=1(i=1, \ldots, 20)$. The matrix $W_{21}^{+}$has an eigenvalue which is quite close to the maximal eigenvalue. The Power method takes 136 iterations to approximate the maximal eigenvalue 10.7461941835 of $W_{21}^{+}$, and 135 iterations for the Iterative algorithm. However, for certain matrices we may obtain fewer iterations by using the test condition $\left|r_{m}^{(k+1)}-r_{m}^{(k)}\right|<\delta$. For example, consider the matrix

$$
A=\left(\begin{array}{lll}
2 & 0 & 1 \\
0.01 & 3 & 0 \\
1 & 0 & 2
\end{array}\right) .
$$

The eigenvalues of $A$ are $3,3,1$. The Iterative algorithm needs only 3 iterations to 
approximate the maximal eigenvalue 3.00000000000 , but more than 1000 iterations, with estimation 3.00230769231, are needed for the Power method to do the job. This is simply because $A$ has double eigenvalue 3 .

Acknowledgement. The author wishes to thank the referee for helpful suggestions which improved the presentation of this paper.

\section{REFERENCES}

1. L. Y. Kolotilina, Lower bounds for the Perron root of a nonnegative matrix, Linear Algebra and Appl. 180 (1993), 133-151.

2. S. L. Liu, Bounds for the greatest characteristic root of a nonnegative matrix, Linear Algebra and Appl. 239 (1996), 151-160.

3. M. Marcus and H. Minc, Modern university algebra (Macmillan, New York, 1965).

4. H. Minc, Nonnegative matrices (John Wiley and Sons, New York, 1988).

5. O. Rojo and R. Jimenez, A decreasing sequence of upper-bounds for the Perron root, Computer Maih. Appl. No 8, 28 (1994), 9-15.

6. J. H. Wilkinson, The algebraic eigenvalue problem (Oxford Univ. Press, 1992).

Department of Mathematics

SOOCHOW UNIVERSITY

TAIPEI

TAIWAN 11102 